\title{
La Sostenibilidad en el Ecuador a Través de un Análisis Multicriterio Basado en Entropía, Durante el Período 2008 - 2015
}

\author{
Escola, Belén 1 iD ; Palma, María-José ${ }^{1}$ iD ; González, Silvia ${ }^{1}$ iD ; Ávalos, Eduardo ${ }^{2, \text { * }}$ (D) \\ ${ }^{1}$ Escuela Politécnica Nacional, Facultad de Ciencias, Quito, Ecuador \\ ${ }^{2}$ Escuela Politécnica Nacional, Facultad de Ingeniería Eléctrica y Electrónica, Quito, Ecuador
}

\begin{abstract}
Resumen: Los programas y políticas implementadas en Ecuador en la última década han permitido el mejoramiento de sus indicadores de Desarrollo Sostenible. Sin embargo, a nivel de país, no se cuenta con estudios que permitan evaluar y dar seguimiento a las 4 dimensiones del Desarrollo Sostenible: económica, social, ambiental e institucional, en conjunto. El Método de Coeficientes Ponderados de Entropía permite analizar de forma objetiva el peso de cada uno de los indicadores, dentro del sistema total y los subsistemas, mediante el grado de diversidad de la información. De esta forma, el análisis de los subsistemas económico, social y ambiental, durante el periodo 2008 - 2015 muestra que el subsistema económico es el que más aporta al Desarrollo Sostenible, y sus indicadores son los de mayor peso en el sistema total. Sin embargo, la evolución de los subsistemas social y ambiental muestra un crecimiento paulatino. Y, en general, un progreso en términos de Desarrollo Sostenible.
\end{abstract}

Palabras clave: sostenibilidad, entropía, análisis multicriterio, teoría de la información.

\section{Sustainability in Ecuador by Means of a Multicriteria Analysis Based on Entropy, Period 2008 - 2015}

\begin{abstract}
Programs and policies implemented during the last decade in Ecuador have improved the sustainable development indicators of the country. However, at the country level, there is a lack of studies related to monitoring and evaluation of the four dimensions of Sustainable Development as a whole: economic, social, environmental and institutional dimension. The Entropy Weighted Coefficient Method enables the objective analysis of the weight of each indicator within the total system and subsystems, through the degree of diversity of information. Accordingly, the analysis of the economic, social and environmental subsystems during the 2008 - 2015 period shows that the economic subsystem is the one that contributes the most to the Sustainable Development, and its indicators have higher relevance within the total system. Nonetheless, the evolution of the social and environmental subsystems shows a gradual growth. In general, this research shows an improvement in terms of Sustainable Development.
\end{abstract}

Keywords: sustainability, entropy, multicriteria analysis, information theory.

\section{INTRODUCCIÓN}

El acuerdo en torno a la búsqueda del Desarrollo a través de la promulgación de los Objetivos de Desarrollo del Milenio (2000 - 2015) y, posteriormente, de los Objetivos de Desarrollo Sostenible (2015 - 2030) muestra la preocupación existente entre los países por alcanzar un desarrollo económico, social y ambiental que garantice el derecho de las futuras generaciones de satisfacer sus propias necesidades.

Para alcanzar un desarrollo sostenible, un país deberá cumplir con las cuatro dimensiones planteadas por Naciones Unidas: económica, social, ambiental e institucional. En el caso de no cumplir con alguna de estas dimensiones, solo se hablará de una sostenibilidad individual para cada uno de los ejes del desarrollo sostenible. Es por esta razón que es fundamental la medición del desarrollo sostenible (Gallopín, 2006, p.9,10).

En virtud de que, a nivel de Ecuador, los estudios sobre el desarrollo sostenible son escasos, esta investigación presenta una medición de la sostenibilidad del Ecuador para el periodo 2008 - 2015, a través del Método de Coeficientes de Peso de la Entropía (EWCM).

El presente artículo se encuentra organizado de la siguiente forma: en la sección 2 se presentan algunos conceptos básicos sobre el Desarrollo Sostenible, la Termodinámica, la Entropía y los indicadores. La sección 3 describe los datos y la metodología empleados. La sección 4 muestra los resultados, totales y por sistema (económico, social, ambiental). Y, la sección 5 expone las conclusiones del estudio.

*f.eduardo.avalos.c@gmail.com

Recibido: 27/03/2020

Aceptado: $23 / 03 / 2021$

Publicado: $31 / 05 / 2021$

$10.33333 /$ rp.vol $47 \mathrm{n} 2.02$

CC BY 4.0 


\section{MARCO TEÓRICO}

\subsection{Desarrollo Sostenible}

En el año 1983 se crea, dentro de la Organización de las Naciones Unidas (ONU), la Comisión de Desarrollo y Medio Ambiente, la misma que brinda un primer concepto de desarrollo sostenible dirigido a todas las naciones:

"Está en manos de la humanidad asegurar que el desarrollo sea sostenible, es decir, asegurar que satisfaga las necesidades del presente sin comprometer la capacidad de las futuras generaciones para satisfacer las propias" (Naciones Unidas, 1997, pág. 55).

A continuación, se presenta un resumen de las reuniones realizadas en el marco de las Naciones Unidas (ONU) con el propósito de que las naciones y la sociedad alcancen el Desarrollo Sostenible; y, el principal acuerdo alcanzado por los mandatarios y organizaciones participantes:

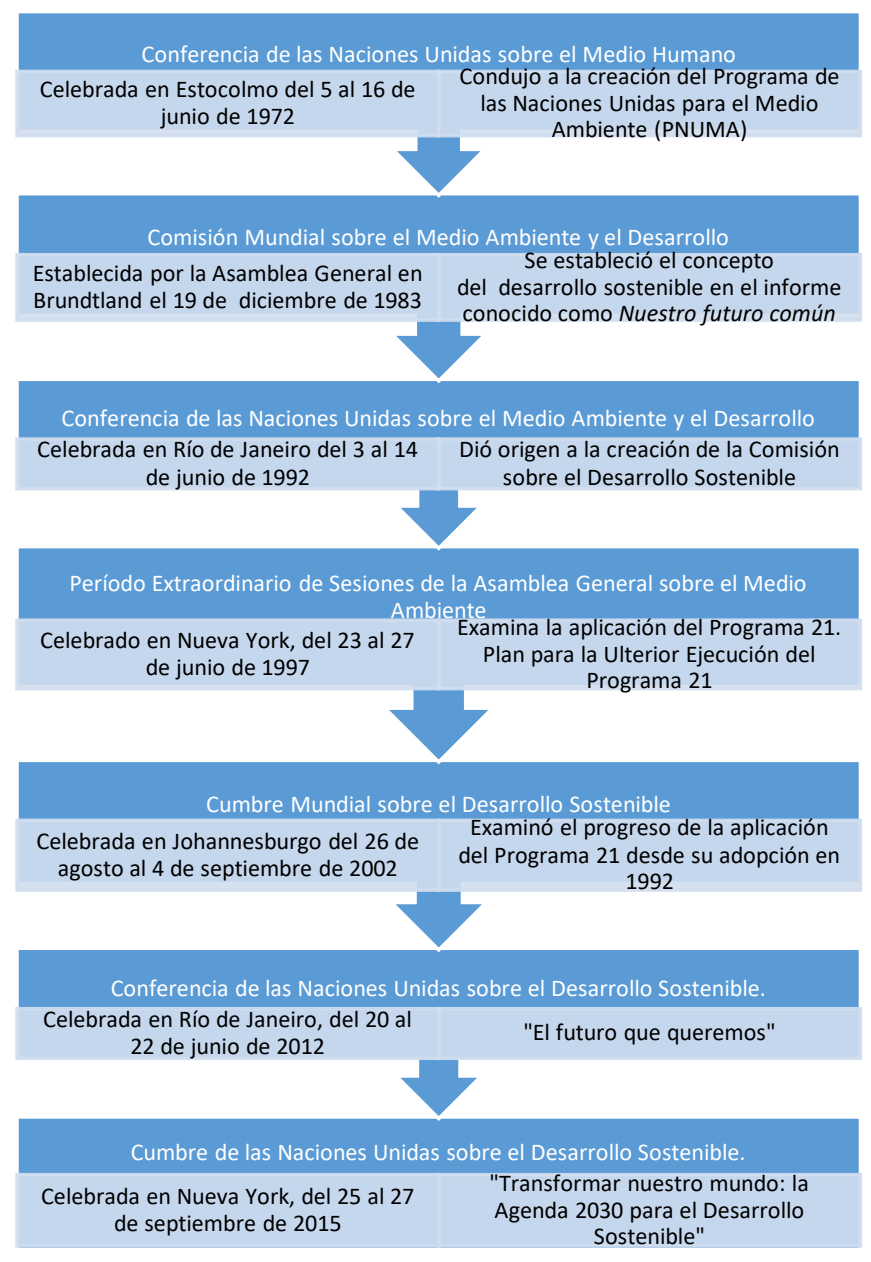

Figura 1. Línea del tiempo sobre el Desarrollo sostenible

Fuente: Naciones Unidas (Martínez, 2018)

Elaborado: Los autores

\subsection{Principios de la Termodinámica}

La termodinámica es la ciencia que estudia las distintas transformaciones energéticas (Müller, 2002). Esta ciencia permite describir matemáticamente los fenómenos que se observan, conocer de mejor manera nuestra vida cotidiana y facilitar el entendimiento de la misma.

La termodinámica tiene dos principales principios: el primero, que establece que la energía no se destruye, pero se transforma. $\mathrm{Y}$, el segundo que nos dice que la materia puede tener una calidad y cantidad dada. Esta segunda ley, conocida como entropía, niega la idea que exista algo espontáneo que transfiera calor de un cuerpo a otro.

\subsection{Sistema}

Un sistema es cualquier colección de materia en la que, en un momento dado, concentremos nuestra atención o se quiere estudiar (Césarman, 1974). Asimismo, se denomina sistema termodinámico a la porción de espacio y su contenido, objeto de nuestro estudio, estando el sistema situado en el interior de una superficie cerrada que lo separa del resto del universo o "medio exterior" (Beléndez, 2017). Es decir, cualquier porción de universo podría ser un sistema.

\subsection{Entropía}

El caos o grado de desorden que existe en la naturaleza se conoce como entropía. Esquemáticamente, la entropía es el progreso para el desorden inherente de un sistema o su destrucción. Según este principio, el Universo se hace más desordenado a cada instante, y existirá un deterioro inexorable y general hacia el caos (Césarman, 1974).

Por otra parte, el concepto de entropía, cercano al de información, y asociado con el caos, tuvo origen en la termodinámica, en cuanto al contenido de información es función del inverso de las probabilidades de los eventos del espacio muestral, y la entropía es el promedio o valor esperado de dicha información (Montenegro, 2011).

\section{DATOS Y METODOLOGÍA}

\subsection{Datos}

Este estudio analiza la información de los indicadores propuestos por la Comisión de Desarrollo Sostenible (CSD) en la guía metodológica de propuesta por Naciones Unidas (United Nations, 2007). Dicha propuesta presenta un conjunto de 96 indicadores que permiten una evaluación amplia del desarrollo sostenible. De éstos, 50 indicadores cumplen tres criterios específicos: 1) cubren temas relevantes para el desarrollo sostenible, 2) proporcionan información crítica; y, 3) son calculados con información disponible en cada país. Estos criterios permiten calificar a los indicadores manejables o base (United Nations, 2007). Los indicadores restantes se denominan indicadores relevantes mismos que son utilizados para ampliar los estudios de desarrollo sostenible.

En el caso de Ecuador se analiza la información de 35 indicadores manejables donde el 74,29\% son indicadores base y el $25,71 \%$ son indicadores relevantes. Así, 6 indicadores corresponden al subsistema ambiental, 12 indicadores al subsistema social, y 17 indicadores son parte del subsistema económico. La información sobre los indicadores en el período 2008-2015 tiene una periodicidad anual y proviene de fuentes como la Comisión Económica para América Latina y el Caribe (CEPAL), el Banco Mundial, el Ministerio del Ambiente, el 
Ministerio de Turismo, el Instituto Nacional de Estadística y Censos (INEC), y el Banco Central del Ecuador (BCE).

\subsection{Metodología}

La toma de decisiones por criterios múltiples se ha incrementado en investigaciones relacionadas a la economía, en los últimos años. Particularmente, con énfasis en investigación operativa y desarrollo sostenible (Zavadskas \& Turskis, 2011).

El principal objetivo de las decisiones multicriterio es el permitir la toma de decisiones a través de las interacciones entre varios factores. Esto permite acercar el análisis a la realidad (Aznar Bellver \& Guijarro Martínez, 2012a).

Simon (1955), analiza la complejidad de las organizaciones actuales, en donde los individuos quieren una toma de decisiones múltiples e incluso incompatibles, buscando la satisfacción de sus necesidades. Moreno- Jiménez (1996, pág. 6) define esta toma de decisiones como:

"Se entiende por decisión multicriterio, el conjunto de aproximaciones, métodos, modelos, técnicas y herramientas dirigidas a mejorar la calidad integral de los procesos de decisión seguidos por los individuos y sistemas, esto es a mejorar la efectividad, eficacia y eficiencia de los procesos de decisión y a incrementar el conocimiento de los mismos (valor añadido del conocimiento)".

El uso de un método polietápico en la ponderación de los factores de decisión sugiere que todos los sistemas; y, por tanto, todos los indicadores, tengan el mismo peso (Tamayo, 2007). Sin embargo, existen varios métodos basados en la teoría de decisión multicriterio. Éstos permiten tener en cuenta aspectos intangibles de los activos a valorar, e inclusive facilitan la incorporación de la experiencia en el proceso. Entre ellos están (Aznar Bellver \& Guijarro Martínez, 2012a):

a) Método CRITIC.

b) Método de la Entropía.

c) Método de la Ordenación simple.

d) Programación por metas.

e) Proceso Analítico Jerárquico, etc.

De esta forma, en lugar de que cada subsistema a analizar (económico, social y ambiental) tenga un peso de 0.33 (Tamayo, 2007); el análisis del peso de los indicadores en el Desarrollo Sostenible para el caso Ecuatoriano emplea el Método de Coeficientes de Peso de la Entropía (EWCM). Este método asigna pesos (ponderación) a los criterios empleados, de manera objetiva, mediante el grado de diversidad de la información.

La aplicación de dicho método requiere la normalización de los datos, puesto que los indicadores poseen diferentes unidades de medida, lo que puede afectar sensiblemente los datos; por lo que se recomienda tener una escala para la comparación (Vafaei et al., 2016).

\subsubsection{Normalización por suma}

Se calcula la contribución del i-ésimo indicador en la j-ésima muestra (años). Así, la normalización por suma de la matriz $\mathrm{X}_{\mathrm{mxn}}^{*}$, se define por la siguiente ecuación:

$$
Y_{\mathrm{ij}}=\frac{x_{i j}^{*}}{\sum_{\mathrm{i}=1}^{\mathrm{m}} x_{i j}^{*}}
$$

La matriz $Y_{i j}$ se presenta como:

$$
Y_{m \times n}=\left[\begin{array}{cccc}
Y_{11} & Y_{12} & \ldots & Y_{1 n} \\
Y_{21} & Y_{22} & \ldots & Y_{2 n} \\
& & & \\
Y_{m 1} & Y_{m 2} & \ldots & Y_{m n}
\end{array}\right]
$$

\subsubsection{Método de la entropía}

La entropía juega un papel importante en la teoría de la información, la cual muestra medidas de información, elección e incertidumbre (Shannon, 1948). La entropía $(H)$ es una formulación de la mecánica estadística. Su fórmula se presenta de la siguiente manera:

$$
H_{i}=-K \sum_{j=1}^{n} a_{i j} \log \left(a_{i j}\right)^{2}
$$

Donde $K$ es una constante positiva y es afectada por el número de los objetos evaluados $(n)$. La constante se determina mediante la fórmula 3:

$$
k=\frac{1}{\log (n)}
$$

La entropía es una medida acotada entre 0 y 1 , es decir $0 \leq$ $H_{i} \leq 1$.

En la teoría de la información, en un sistema donde existe un desorden total, la entropía es máxima, es decir $H_{i}=1$ todos los estados posibles son equiprobables. Mientras que en un sistema con un orden total la entropía es igual a 0 , por tanto existe 0 incertidumbre (Montenegro, 2011).

Para la obtención de $a_{i j}$ se define la siguiente ecuación (4), que también es una normalización por suma, la cual indica la contribución del i-ésimo indicador en el j-ésimo año (Aznar Bellver \& Guijarro Martínez, 2012c):

$$
a_{i j}=\frac{d_{i j}}{\sum_{i=1}^{m} d_{i j}}
$$

Donde $\mathrm{a}_{\mathrm{ij}}$ equivale a una proporción donde $\sum a_{i j}=1$ y $0 \leq$ $a_{i j} \leq 1$

Los valores para utilizar en el método de entropía se representan como se muestra en la siguiente matriz:

\footnotetext{
${ }^{2}$ (Aznar Bellver \& Guijarro Martínez, 2012b)
} 


$$
\left[a_{i j}\right]=\left[\begin{array}{cccc}
a_{11} & a_{12} & \cdots & a_{1 n} \\
a_{21} & \ddots & \cdots & \vdots \\
\vdots & \vdots & \ddots & \vdots \\
a_{m 1} & \cdots & \cdots & a_{m n}
\end{array}\right]
$$

El verdadero valor del indicador denominado variación de la evaluación del indicador depende de la diferencia entre el coeficiente $D_{i}$ y 1(Wang et al., 2015), que se obtiene mediante la ecuación (5):

$$
D_{i}=1-H_{i}
$$

En el método de la entropía, si el valor de la ecuación (5) es baja (cercana a 0 ), significa que la variación de la evaluación del indicador es alta, es decir, el indicador es importante. Y, en el caso contrario, el indicador reflejará poca importancia (Zhao et al., 2017).

Finalmente, se normaliza $D_{i}$ mediante normalización por la suma $^{3}$ y se obtienen las ponderaciones para cada criterio o indicador. Para determinar los pesos o ponderación de los indicadores se establece la ecuación (6).

$$
w_{i}=\frac{D_{i}}{\sum_{i=1}^{m} D_{i}}
$$

Donde $w_{j}$ debe satisfacer la condición $0 \leq w_{i} \leq 1 y$ $\sum_{i=1}^{m} w_{i}=1$.

De esta forma, el método aplicado permite evaluar en primera instancia la normalización de los datos. Seguido con el planteamiento de la entropía de la información, donde se determinan los pesos de cada indicador.

Para la evaluación del Desarrollo Sostenible, a nivel de sistema y subsistema, Wang (2015) plantea un indicador que este trabajo denomina Indicador de Desarrollo Sostenible (IDS) y se obtiene mediante la ecuación (7):

$$
f_{j}=\sum_{i=1}^{m} f_{i j}
$$

El aumento de este indicador muestra un mayor Desarrollo Sostenible, y viceversa. Definido como Índice Multicriterio mediante la ecuación (8):

$$
f_{i j}=w_{i} * d_{i j}
$$

Donde $d_{i j}$ son los valores obtenidos por mínimos y máximos.

\section{RESULTADOS Y DISCUSIÓN}

\subsection{Resultados del Sistema General}

En la Tabla 1 se pueden apreciar los pesos de los indicadores

\begin{tabular}{|c|c|c|c|}
\hline Indicador & Peso & Indicador & Peso \\
\hline PIB per cápita & 0,04 & $\begin{array}{l}\text { Pobreza extrema } \\
\text { nacional }\end{array}$ & 0,03 \\
\hline Formación bruta de capital & 0,02 & Saneamiento & 0,02 \\
\hline Ahorro neto ajustado & 0,02 & Fuente de agua mejorada & 0,03 \\
\hline Tasa de inflación & 0,01 & Hogares sin electricidad & 0,02 \\
\hline Saldo de la deuda externa & 0,03 & Homicidios intencionales & 0,05 \\
\hline Tasa de empleo bruta & 0,04 & $\begin{array}{l}\text { Tasa de mortalidad de } \\
\text { menores de cinco años }\end{array}$ & 0,02 \\
\hline Empleo vulnerable & 0,03 & La esperanza de vida & 0,03 \\
\hline Uso de Internet & 0,02 & $\begin{array}{l}\text { Tasa neta de matrícula } \\
\text { en educación primaria }\end{array}$ & 0,02 \\
\hline Telefonía fija & 0,02 & $\begin{array}{l}\text { Nivel de escolaridad } \\
\text { secundaria de adultos }\end{array}$ & 0,03 \\
\hline Telefonía celular móvil & 0,03 & $\begin{array}{l}\text { Tasa de alfabetización de } \\
\text { adultos }\end{array}$ & 0,03 \\
\hline Turismo/PIB & 0,03 & $\begin{array}{l}\text { Crecimiento de la } \\
\text { población }\end{array}$ & 0,03 \\
\hline Saldo en cuenta corriente & 0,04 & $(\mathrm{CO} 2)$, per cápita & 0,03 \\
\hline Inversión extranjera directa & 0,03 & Consumo (SAO) & 0,03 \\
\hline Remesas de trabajadores & 0,05 & Tierra agrícola & 0,03 \\
\hline $\begin{array}{l}\text { Energía renovable del total } \\
\text { de consumo final de energía }\end{array}$ & 0,03 & $\begin{array}{l}\text { Área de tierra cubierta } \\
\text { por bosques }\end{array}$ & 0,03 \\
\hline Intensidad energética & 0,02 & Área marina protegida & 0,03 \\
\hline Consumo de energía & 0,02 & Área terrestre protegida & 0,02 \\
\hline Pobreza nacional & 0,03 & & \\
\hline
\end{tabular}
de Desarrollo Sostenible del sistema general o total. Estos se encuentran entre $1,62 \%$ y $4,84 \%$. De esta forma, se pueden comparar los resultados con los del método polietápico ${ }^{4}$.
Tabla 1. Pesos de los Indicadores de Desarrollo Sostenible en el Sistema

Como se observa en la Figura 2, al aplicar el Método de Coeficientes de Peso de la Entropía (EWCM), ordenados de mayor a menor, los tres mejores años en cuanto a Desarrollo Sostenible ecuatoriano son 2014, 2015 y 2013. Los mismos resultados son válidos al emplear una media ponderada en los indicadores. Mientras que, al aplicar el método polietápico, el orden de los mejores años para el Desarrollo Sostenible en Ecuador son los años 2015, 2014 y 2013.

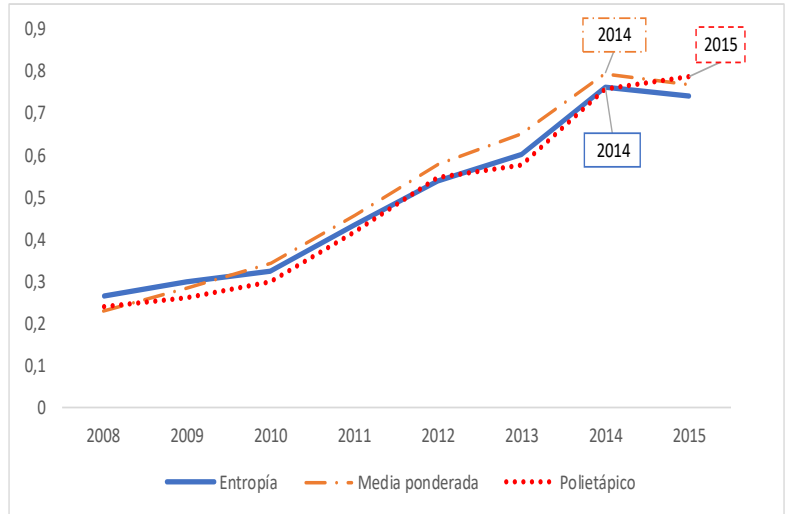

Figura 2. Evolución del sistema considerando los pesos de los indicadores

Según Gallopín (2006), un país tendrá un Desarrollo Sostenible cuando cumpla con todas las dimensiones que lo componen. En tal virtud, sería deseable que las tres dimensiones analizadas (económica, social y ambiental) posean un valor igual. Sin embargo, los resultados muestran que el Subsistema Ambiental demanda mayor intervención

\footnotetext{
${ }^{4}$ Método que sugiere que todos los sistemas tengan el mismo peso.
} 
para alcanzar el nivel de referencia, a pesar de los avances logrados.

\subsection{Sistema Económico}

En la Tabla 2 se muestra que las cuestiones relacionadas con el Desarrollo Económico, a través del EWCM, superan el peso determinado por el Método polietápico. Al contrario de lo que ocurre con la asociación económica global y el patrón de consumo y producción.

Esto es el reflejo de una economía ecuatoriana sustentada en la producción de materias primarias, mayoritariamente. Por ejemplo, históricamente, la economía del país ha estado marcada por la exportación de productos como el cacao, el banano y el petróleo. Además, las medidas adoptadas por los Estados Unidos y Europa ante la crisis del año 2018 trajeron consecuencias para todos los países con los que tenían negocios (Acosta \& Serrano, 2009), Ecuador entre ellos.

Tabla 2. Pesos de temas subtemas e indicadores del subsistema económico

\begin{tabular}{|c|c|c|c|c|}
\hline Temas & Subtemas & Pesos & Indicadores & Pesos \\
\hline \multirow{16}{*}{$\begin{array}{l}\text { Desarrollo } \\
\text { Económico. } \\
0,60 \\
{[0,33]}\end{array}$} & \multirow{6}{*}{$\begin{array}{l}\text { Desempeño } \\
\text { Macroeconómico. }\end{array}$} & \multirow{6}{*}{$\begin{array}{r}0,181 \\
{[0,667]}\end{array}$} & \multirow{2}{*}{ PIB per cápita. } & 0,759 \\
\hline & & & & {$[0,167]$} \\
\hline & & & $\begin{array}{l}\text { Formación bruta de } \\
\text { capital. }\end{array}$ & $\begin{array}{r}0,413 \\
0,1671\end{array}$ \\
\hline & & & \multirow{3}{*}{$\begin{array}{l}\text { Ahorro neto } \\
\text { ajustado. } \\
\text { Tasa de inflación. }\end{array}$} & 0,336 \\
\hline & & & & {$[0,167]$} \\
\hline & & & & $\begin{array}{r}0,297 \\
{[0,167]}\end{array}$ \\
\hline & \multirow{4}{*}{$\begin{array}{l}\text { Finanzas Públicas } \\
\text { Sostenibles. } \\
\text { Empleo. }\end{array}$} & 0,544 & \multirow{4}{*}{$\begin{array}{l}\text { Saldo de la deuda } \\
\text { externa. } \\
\text { Tasa de empleo } \\
\text { bruta. } \\
\text { Empleo vulnerable. }\end{array}$} & 0,544 \\
\hline & & {$[0,667]$} & & {$[0,667]$} \\
\hline & & $\begin{array}{r}0,145 \\
{[0,667]}\end{array}$ & & $\begin{array}{r}0,799 \\
{[0,333]}\end{array}$ \\
\hline & & & & $\begin{array}{r}0,651 \\
{[0,333]}\end{array}$ \\
\hline & \multirow{5}{*}{$\begin{array}{l}\text { Información y } \\
\text { tecnología de } \\
\text { comunicación. }\end{array}$} & \multirow{5}{*}{$\begin{array}{r}0,159 \\
{[0,667]}\end{array}$} & \multirow{5}{*}{$\begin{array}{l}\text { Personas que usan } \\
\text { Internet. } \\
\text { Suscripciones a } \\
\text { telefonía fija. } \\
\text { Suscripciones a } \\
\text { telefonía celular } \\
\text { móvil. }\end{array}$} & 0,501 \\
\hline & & & & {$[0,222]$} \\
\hline & & & & $\begin{array}{r}0,474 \\
{[0,222]}\end{array}$ \\
\hline & & & & 0,614 \\
\hline & & & & {$[0,222]$} \\
\hline & Turismo. & $\begin{array}{r}0,599 \\
{[0,667]}\end{array}$ & $\begin{array}{l}\text { Contribución del } \\
\text { turismo en el PIB. }\end{array}$ & $\begin{array}{r}0,599 \\
{[0,667]}\end{array}$ \\
\hline \multirow{4}{*}{$\begin{array}{l}\text { Asociación } \\
\text { económica } \\
\text { global. } 0,24 \\
{[0,33]}\end{array}$} & Comercio. & $\begin{array}{r}0,820 \\
{[0,167]}\end{array}$ & $\begin{array}{l}\text { Saldo en cuenta } \\
\text { corriente. }\end{array}$ & $\begin{array}{r}0,820 \\
{[0,1667]}\end{array}$ \\
\hline & \multirow{3}{*}{$\begin{array}{l}\text { Financiamiento } \\
\text { externo. }\end{array}$} & 0,163 & \multirow{2}{*}{$\begin{array}{l}\text { Inversión extranjera } \\
\text { directa. }\end{array}$} & 0,577 \\
\hline & & {$[0,167]$} & & {$[0,833]$} \\
\hline & & & $\begin{array}{l}\text { Remesas de } \\
\text { trabajadores. }\end{array}$ & $\begin{array}{r}0,105 \\
{[0,833]}\end{array}$ \\
\hline \multirow{7}{*}{$\begin{array}{l}\text { Patrón de } \\
\text { consumo y } \\
\text { producción. } \\
0,16 \\
{[0,33]}\end{array}$} & \multirow[t]{7}{*}{ Uso de la energía. } & \multirow{7}{*}{$\begin{array}{r}0,157 \\
{[0,33]}\end{array}$} & \multirow{4}{*}{$\begin{array}{l}\text { Porcentaje de } \\
\text { energía renovable } \\
\text { respecto al total de } \\
\text { consumo final de } \\
\text { energía. }\end{array}$} & 0,664 \\
\hline & & & & {$[0,111]$} \\
\hline & & & & \\
\hline & & & & \\
\hline & & & Intensidad & 0,426 \\
\hline & & & energética. & {$[0,111]$} \\
\hline & & & Consumo de & 0,476 \\
\hline
\end{tabular}

[ ] Pesos obtenidos mediante el método Polietápico.

Hacia el año 2009, Ecuador implementó políticas económicas y sociales para atenuar la crisis. Entre ellas, el establecimiento de estímulos fiscales para la obtención de préstamos y el aumento del gasto en educación y salud (Ray \& Kozameh, 2012).

Ese año se registra un decrecimiento debido a la baja de los ingresos nominales de las exportaciones petroleras en un $41 \%$, aproximadamente. Pero, se puede observar un ascenso en la exportación de productos no petroleros por lo que la economía total no tiene un declive notorio (Banco Central del Ecuador, 2012).

Al no ser una economía con moneda propia, Ecuador puso como medida de defensa, salvaguardias por balanzas de pagos. Éstas afectaron inclusive a los países con los que se tienen acuerdos comerciales (CEPAL-Naciones Unidas, 2009). De esta forma, adicional al arancel nacional, se aplicaron un recargo ad-valorem (entre el $30 \%$ y el $35 \%$ ) a productos del tipo: azucarados, confitería, bebidas alcohólicas, artículos electrónicos, juguetes y otros. Además, un recargo específico (entre 10 y 12 dólares por unidad de medida) a productos correspondientes a los sectores: textil, calzado y cerámica. Y, se establecieron cuotas, limitando el valor de las importaciones de mercancías (a un cupo entre $65 \%$ y $70 \%$ de CIF $^{5}$ ) en productos como galletas, preparados y congelados alimenticios varios, perfumes y aguas de tocador, entre otros (COMEXI, 2009).

Por su parte, las características de la formación bruta de capital, el consumo privado y las exportaciones en el año 2010, determinaron los pesos más bajos en el sistema económico. Además, ese mismo año, Ecuador registró una deflación relacionada con la política de promoción de precios de la oferta y la provisión de prodeuctos agrícolas; y, mayores tasas de empleo (Banco Central del Ecuador, 2010).

En el año 2011 existió un crecimiento determinado, en gran parte, por el aumento acelerado en el sector de construcción que abarcará alrededor del $40 \%$ del Producto Interno Bruto (PIB) total. Crecimiento relacionado con la existencia de programas de vivienda y préstamos hipotecarios (Ray \& Kozameh, 2012).

Posteriormente, en el año 2012, el gasto público aumentó; y, también, la deuda externa. Esto puso en riesgo la Balanza de pagos con un saldo en cuenta corriente que permanecía negativo.

En 2013, Ecuador tuvo un crecimiento en PIB de 4,5\%, logrando ubicarse entre los mejores resultados económicos de América del Sur. Este crecimiento estuvo determinado por las exportaciones de productos no petroleros, que representaron para dicho año el 85,4\% de total del PIB (Banco Central del Ecuador, 2014).

La economía ecuatoriana en el año 2014 se posicionó entre las 4 mejores del sur del continente americano, con un crecimiento de 3,8\% en el PIB; superando a Perú, México y Chile. Este crecimiento estuvo definido por el crecimiento del gasto de consumo final de los hogares, las exportaciones y las inversiones.

Para el año 2015, la economía ecuatoriana tuvo un crecimiento de $0,2 \%$ respecto al año anterior, esto gracias al gasto de consumo final del gobierno general, mientras que la formación bruta de capital tuvo una caída. Pero, la inversión privada creció, justificada por la adquisición de activos fijos. Sin

\footnotetext{
${ }^{5}$ CIF (acrónimo del término en inglés Cost, Insurance and Freight, «Coste, seguro y flete, puerto de destino convenido»)
} 
olvidar que, a partir del año 2014, el dólar sufrió una depreciación, y el precio de petróleo bajó.

\subsection{Subsistema Social}

Dentro del subsistema social, los temas de pobreza y educación tienen un mayor aporte: 39,32\% y 23,14\%, respectivamente. Cuestiones como Gobernanza 14,19\%, Salud $14,79 \%$ y Demografía $8,56 \%$, también se pueden apreciar en la Tabla 3.

Tabla 3. Pesos de temas subtemas e indicadores del subsistema Social

\begin{tabular}{|c|c|c|c|c|}
\hline Temas & Subtemas & Pesos & Indicadores & Pesos \\
\hline \multirow[t]{5}{*}{$\begin{array}{l}\text { Pobreza. } \\
0,393 \\
{[0,20]}\end{array}$} & $\begin{array}{l}\text { Pobreza de } \\
\text { ingresos. }\end{array}$ & $\begin{array}{l}0,179 \\
{[0,5]}\end{array}$ & $\begin{array}{l}\text { Proporción de la } \\
\text { población que vive por } \\
\text { debajo del umbral de } \\
\text { pobreza nacional. }\end{array}$ & $\begin{array}{r}0,888 \\
{[0,25]}\end{array}$ \\
\hline & & & $\begin{array}{l}\text { Proporción de la } \\
\text { población que vive por } \\
\text { debajo del umbral de } \\
\text { pobreza extrema } \\
\text { nacional. }\end{array}$ & $\begin{array}{r}0,906 \\
{[0,25]}\end{array}$ \\
\hline & Saneamiento. & $\begin{array}{l}0,717 \\
{[0,5]}\end{array}$ & $\begin{array}{l}\text { Proporción de la } \\
\text { población que utiliza } \\
\text { una instalación de } \\
\text { saneamiento mejorada. }\end{array}$ & $\begin{array}{l}0,717 \\
{[0,5]}\end{array}$ \\
\hline & Agua potable. & $\begin{array}{c}0,735 \\
{[0,5]}\end{array}$ & $\begin{array}{l}\text { Proporción de la } \\
\text { población que utiliza } \\
\text { una fuente de agua } \\
\text { mejorada. }\end{array}$ & $\begin{array}{r}0,735 \\
{[0,5]}\end{array}$ \\
\hline & $\begin{array}{l}\text { Acceso a la } \\
\text { energía. }\end{array}$ & $\begin{array}{l}0,687 \\
{[0,5]}\end{array}$ & $\begin{array}{l}\text { Proporción de hogares } \\
\text { sin electricidad u otros } \\
\text { servicios modernos de } \\
\text { energía. }\end{array}$ & $\begin{array}{r}0,687 \\
{[0,5]}\end{array}$ \\
\hline $\begin{array}{l}\text { Gobernanza. } \\
0,1419 \\
{[0,20]}\end{array}$ & Crimen. & $\begin{array}{r}0,142 \\
{[0,20]}\end{array}$ & $\begin{array}{l}\text { Número de homicidios } \\
\text { intencionales. }\end{array}$ & $\begin{array}{r}0,1419 \\
{[0,20]}\end{array}$ \\
\hline $\begin{array}{l}\text { Salud. } \\
0,1479 \\
{[0,20]}\end{array}$ & Mortalidad. & $\begin{array}{r}0,1479 \\
{[0,20]}\end{array}$ & $\begin{array}{l}\text { Tasa de mortalidad de } \\
\text { menores de cinco años. } \\
\text { La esperanza de vida al } \\
\text { nacer. }\end{array}$ & $\begin{array}{r}0,664 \\
{[0,10]} \\
0,815 \\
{[0,10]}\end{array}$ \\
\hline \multirow[t]{2}{*}{$\begin{array}{l}\text { Educación. } \\
0,231 \\
{[0,20]}\end{array}$} & $\begin{array}{l}\text { Nivel de } \\
\text { Educación. }\end{array}$ & $\begin{array}{r}0,1565 \\
{[0,10]}\end{array}$ & $\begin{array}{l}\text { Tasa neta de matrícula } \\
\text { en educación primaria. } \\
\text { Nivel de escolaridad } \\
\text { secundaria de adultos. }\end{array}$ & $\begin{array}{l}0,626 \\
{[0,5]} \\
0,938 \\
{[0,5]}\end{array}$ \\
\hline & Alfabetismo. & $\begin{array}{r}0,749 \\
{[0,10]}\end{array}$ & $\begin{array}{l}\text { Tasa de alfabetización } \\
\text { de adultos. }\end{array}$ & $\begin{array}{r}0,749 \\
{[0,10]}\end{array}$ \\
\hline $\begin{array}{l}\text { Demografía. } \\
0,856 \\
{[0,20]}\end{array}$ & Población. & $\begin{array}{r}0,856 \\
{[0,20]}\end{array}$ & $\begin{array}{l}\text { Crecimiento de la } \\
\text { población. }\end{array}$ & $\begin{array}{r}0,856 \\
{[0,20]}\end{array}$ \\
\hline
\end{tabular}

[ ] Pesos obtenidos mediante el método Polietápico.

A partir del año 2009, indicadores como la proporción de la población que vive por debajo del umbral de pobreza nacional y la proporción de la población que vive por debajo del umbral de pobreza extrema nacional, disminuyeron. Esto se ha atribuido, en parte, a programas como el Bono de Desarrollo Humano.

Otra de las medidas adoptadas fue el impulso a la revolución productiva, del trabajo y el empleo. Y, a través de los años, la planificación estuvo a cargo de instituciones como la Secretaría Nacional de Planificación y Desarrollo (SENPLADES), el Ministerio de Agricultura y Ganadería (MAGAP), el Ministerio de Inclusión Económica y Social (MIESS), entre otras.

Entre las medidas para erradicar la pobreza, el proporcionar servicios básicos a la población es prioritario. Indicadores como la proporción de la población que utiliza una instalación de saneamiento mejorada y proporción de la población que utiliza una fuente de agua mejorada han aumentado, mientras que el indicador proporción de hogares sin electricidad $\mathrm{u}$ otros servicios modernos de energía ha disminuido.

Para la sociedad, uno de los temas que genera mayor preocupación es el de criminalidad. Este se considera para el cálculo del peso del Subsistema Social. Así, el número de homicidios intencionales por cada 100.000 habitantes ha disminuido en un $11,5 \%$ en el periodo de estudio.

En lo relativo a la salud, la Constitución ecuatoriana especifica que ésta es rol y responsabilidad del Estado, el cual garantizará su universalidad y gratuidad. Este estudio emplea indicadores como tasa de mortalidad de menores de cinco años y la esperanza de vida al nacer en la medición de la salud.

En el año 2010, se aprobó la Ley Orgánica de Participación Ciudadana. Ésta tuvo repercusión en el reconocimiento de los derechos de la sociedad. A partir de ella, se dividieron las competencias en salud para un mejor control y cumplimiento, dando papeles a cada uno de los siguientes sistemas:

- Nivel central: políticas

- Gobiernos municipales: Planificación, construcción y mantenimiento infraestructural de las casas de salud.

- Distritos Metropolitanos Autónomos: Planificación, construcción y mantenimiento infraestructural de las casas de salud.

Con el pasar de los años se han implementado distintos programas de salud pública para la prevención de enfermedades y el cuidado de los más pequeños; y, la garantía del cuidado prenatal. Entre las iniciativas implementadas se encuentran:

- Desnutrición cero.

- Modelo de Atención Integral de Salud.

- Atención Primaria de Salud Renovada.

- "Ecuador saludable, Vuelvo a ti", entre otros.

Además, el gobierno ecuatoriano se enfocó, en gran parte, en la educación. La mejora en este Subsistema se dio a través de la alfabetización de la población y la educación gratuita. Los indicadores: nivel de escolaridad secundaria de adultos (terciaria) y tasa de alfabetización de adultos aumentaron. Así, la tasa de alfabetización en adultos llegó al 99,1\%, en 2015.

\subsection{Sistema Ambiental}

Ecuador fue parte de la primera reunión en la que se planteó el Desarrollo Sostenible en el año 1972. Este sería el punto de partida para la concepción de un desarrollo amigable con el Medio Ambiente. Sin embargo, al ser un país productor de materias primas, las medidas implementadas no podían ser muy efectivas, sobre todo, por la dependencia económica del país de las extracciones de petróleo, las minas y la explotación agrícola.

A partir del año 2000, el país se comprometió con el cumplimiento de las metas planteadas por los Objetivos de Desarrollo del Milenio (ODM), para lograr un desarrollo económico, social y ambiental, a la vez. Además, en el año 
2008, Ecuador constitucionalizó al Medio Ambiente, lo que propició el crecimiento del Subsistema Ambiental.

\begin{tabular}{|c|c|c|c|c|}
\hline Temas & Subtemas & Pesos & Indicadores & Pesos \\
\hline \multirow[t]{2}{*}{$\begin{array}{l}\text { Atmósfera. } \\
0,3133 \\
{[0,25]}\end{array}$} & $\begin{array}{l}\text { Cambio } \\
\text { climático. }\end{array}$ & $\begin{array}{r}0,1705 \\
{[0,125]}\end{array}$ & $\begin{array}{l}\text { Emisiones de } \\
\text { dióxido de } \\
\text { carbono. }\end{array}$ & $\begin{array}{r}0,1705 \\
{[0,125]}\end{array}$ \\
\hline & $\begin{array}{l}\text { Agotamiento } \\
\text { de la capa de } \\
\text { ozono. }\end{array}$ & $\begin{array}{r}0,1429 \\
{[0,125]}\end{array}$ & $\begin{array}{l}\text { Consumo de } \\
\text { sustancias que } \\
\text { agotan la capa } \\
\text { de ozono. }\end{array}$ & $\begin{array}{r}0,1429 \\
{[0,125]}\end{array}$ \\
\hline \multirow{2}{*}{$\begin{array}{l}\text { Tierra. } \\
0,354 \\
{[0,25]}\end{array}$} & Agricultura. & $\begin{array}{r}0,1838 \\
{[0,125]}\end{array}$ & $\begin{array}{l}\text { Área cultivable } \\
\text { y de cultivo }\end{array}$ & $\begin{array}{r}0,1838 \\
{[0,125]}\end{array}$ \\
\hline & Bosques. & $\begin{array}{r}0,1698 \\
{[0,125]}\end{array}$ & $\begin{array}{l}\text { permanente. } \\
\text { Proporción del } \\
\text { área de tierra } \\
\text { cubierta por } \\
\text { bosques. }\end{array}$ & $\begin{array}{r}0,1698 \\
{[0,125]}\end{array}$ \\
\hline $\begin{array}{l}\text { Océanos, mares y } \\
\text { costas. } \\
0,193 \quad[0,25]\end{array}$ & $\begin{array}{l}\text { Ambiente } \\
\text { marino. }\end{array}$ & $\begin{array}{l}0,1934 \\
{[0,25]}\end{array}$ & $\begin{array}{l}\text { Proporción de } \\
\text { área marina } \\
\text { protegida. }\end{array}$ & $\begin{array}{r}0,1934 \\
{[0,25]}\end{array}$ \\
\hline $\begin{array}{l}\text { Biodiversidad. } \\
0,1396 \\
{[0,25]}\end{array}$ & Ecosistema. & $\begin{array}{r}0,1396 \\
{[0,25]}\end{array}$ & $\begin{array}{l}\text { Proporción de } \\
\text { área terrestre } \\
\text { protegida, total } \\
\text { y por región } \\
\text { ecológica. }\end{array}$ & $\begin{array}{r}0,1396 \\
{[0,25]}\end{array}$ \\
\hline
\end{tabular}

[ ] Pesos obtenidos mediante el método Polietápico.

La Tabla 4 muestra los indicadores del subsistema ambiental. Uno de los principales indicadores para la evaluación de este Subsistema es la emisión de dióxido de carbono per cápita. No se evidencia una disminución notable de este valor durante el período de estudio.

Por su parte, el indicador sobre el consumo de sustancias agotadoras de la capa de ozono ha disminuido 59.7\%. Entre las razones de esta reducción se encuentran programas como:

- Plan Nacional de Espumas.

- Restricción del uso de bromuro de metilo, sustancia agotadora de la Capa de Ozono, para fines agrícolas.

- Plan Renova Refrigeradora durante 2013 - 2016.

- Conservación del Yasuní.

- Cambio de cocinas de gas a inducción, entre otros.

Por otra parte, el indicador sobre la proporción de área marina protegida ha aumentado en el periodo de estudio en un $34 \%$, esto gracias a las políticas tomadas para el cuidado de flora y fauna marina, sobre todo en la Región Insular.

El indicador relacionado con la tierra agrícola experimentó una disminución. Esto sería favorable para la conservación del Medio Ambiente ya que reflejaría una producción agrícola más consciente en un país de producción primaria.

Para poder disminuir el impacto de la deforestación y la expansión de la frontera agrícola, Ecuador implementó programas como: "Programa Socio - Bosque" y el "Programa Nacional de Forestación y Reforestación”; con el objetivo principal de reforestar 1 millón de hectáreas en 20 años (Ministerio del Ambiente del Ecuador, 2011).

Finalmente, los indicadores sobre la proporción del área de tierra cubierta por bosques y la proporción de área terrestre protegida no muestran cambios llamativos. Esto obedece, en gran parte, a que el país cuenta con protección a sus bosques y áreas protegidas desde mucho antes del periodo de estudio.

\section{CONCLUSIONES}

Este artículo muestra la situación de Ecuador en materia de Desarrollo Sostenible de una manera objetiva. Además, constituye una base informada para el fomento de cada dimensión del Desarrollo Sostenible.

La aplicación del Método de Coeficientes Ponderados de Entropía (EWCM) es una deducción de la teoría de la información. De esta forma, aquellos indicadores que muestran una alta variación, presentan mayor nivel de información implementada en un evento (política o programa) determinado. Caso contrario, cuando la información es deficitaria o inexistente, los eventos serán equiprobables.

Del análisis se desprende que, el peso que tiene el Subsistema Económico es mayor que el de los sistemas Social y Ambiental en la consecución de un Desarrollo Sostenible. Los logros del subsistema sistema económico se reducen hasta el año 2015, mientras mejoran los resultados de los subsistemas Social y Ambiental. Esto da una visión general de la búsqueda del Desarrollo Sostenible, brindando mayor importancia a la naturaleza y al ser humano.

Durante el periodo analizado, dentro del subsistema Social, Ecuador brindó mayor atención a áreas como: la educación, la salud, y la equidad y redistribución de la riqueza. Estos temas alcanzaron una mayor ponderación dentro de dicho Subsistema.

Por su parte, el Subsistema Ambiental refleja que gran parte de los bosques y páramos se encuentran amenazados por la minería industrial y la extracción de materias primas. Sin embargo, Ecuador eleva a interés público la preservación del ambiente y, por tanto, la preservación de la flora y fauna.

\section{REFERENCIAS}

Acosta, A., \& Serrano, A. (2009). Ecuador frente a la crisis económica. 17.

Aznar Bellver, J., \& Guijarro Martínez, F. (2012a). Nuevos métodos de valoración: Modelos multicriterio. Universitat Politècnica de València.

Banco Central del Ecuador. (2010, junio). Evolución de la Economía Ecuatoriana. https://contenido.bce.fin.ec/documentos/Estadisticas /SectorReal/Previsiones/IndCoyuntura/EvolucionEc onEcu_06-10.pdf

Banco Central del Ecuador. (2014, abril 19). La economía ecuatoriana tuvo un crecimiento anual de $4.5 \%$ en 2013.

https://www.bce.fin.ec/index.php/archivo/item/623la-econom\%C3\%ADa-ecuatoriana-tuvo-uncrecimiento-anual-de-45-en-2013

Banco Central del Ecuador, D. G. de E. (2012). La Economía Ecuatoriana Luego de 10 años de Dolarización. https://contenido.bce.fin.ec/documentos/Publicacion 
esNotas/Notas/Dolarizacion/Dolarizacion10anios.pd f

Beléndez, A.

(2017).

Termodinámica. https://rua.ua.es/dspace/bitstream/10045/95267/1/FF I-I-Termodinamica.pdf

CEPAL-Naciones Unidas. (2009). Estudio económico de América Latina y el Caribe.

COMEXI (2009). Resolución 466. http://intranet.comunidadandina.org/Documentos/DI nformativos/SGdi914.pdf

Césarman, E. (1974). Hombre y entropía (Editorial PaxMéxico).

Gallopín, G. C. (2006). Sostenibilidad del Desarrollo en América Latina y el Caribe: Cifras y tendencias Honduras. 51.

Martínez, M. (2018, diciembre). Documentación de la ONU: Medio ambiente [Guía de investigación]. //research.un.org/es/docs/environment/conferences

Ministerio del Ambiente del Ecuador. (2011). Segundo informe nacional para el convenio sobre la diversidad biológica. 177.

Montenegro, Á. (2011). Información Y Entropía En Economía. Revista de Economía Institucional, 13(25), 23.

Moreno-Jiménez. (1996). Metodología Multicriterio en el Plan Nacional de Regadios.

Müller, E. A. (2002). Termodinámica básica. Kemiteknik.

Naciones Unidas. (1997, septiembre 19). Resolución aprobada por la asamblea general. https://undocs.org/es/A/RES/S-19/2

Ray, R., \& Kozameh, S. (2012). La economía de Ecuador desde 2007. http://cepr.net/documents/publications/ecuadorespanol-2012-05.pdf

Shannon, C. E. (1948). A Mathematical Theory of Communication. Bell System Technical Journal, 27(3), 379-423. https://doi.org/10.1002/j.15387305.1948.tb01338.x

SIMON H. (1955). A Behavior Model of Rational Choice. Quarterly Journal of Economics.

Tamayo, G. (2007). Diseños muestrales en la investigación. 4(7), 14.

United Nations (Ed.). (2007). Indicators of sustainable development: Guidelines and methodologies (3rd ed). United Nations.
Vafaei, N., Ribeiro, R. A., \& Camarinha-Matos, L. M. (2016). Normalization Techniques for Multi-Criteria Decision Making: Analytical Hierarchy Process Case Study. En L. M. Camarinha-Matos, A. J. Falcão, N. Vafaei, \& S. Najdi (Eds.), Technological Innovation for Cyber-Physical Systems (Vol. 470, pp. 261-269). Springer International Publishing. https://doi.org/10.1007/978-3-319-31165-4_26

Wang, Q., Yuan, X., Zhang, J., Gao, Y., Hong, J., Zuo, J., \& Liu, W. (2015). Assessment of the Sustainable Development Capacity with the Entropy Weight Coefficient Method. Sustainability, 7(10), 13542 13563. https://doi.org/10.3390/su71013542

Zavadskas, E. K., \& Turskis, Z. (2011). Multiple Criteria Decision Making (Mcdm) Methods in Economics: An Overview / Daugiatiksliai Sprendimų Prièmimo Metodai Ekonomikoje: Apžvalga. Technological and Economic Development of Economy, 17(2), 397-427. https://doi.org/10.3846/20294913.2011.593291

Zhao, X., Guo, H.-T., Huang, C.-L., \& Zhong, J.-S. (2017). Teaching evaluation system research based on structure entropy weight method. Journal of Discrete Mathematical Sciences and Cryptography, 20(1), 179-191. https://doi.org/10.1080/09720529.2016.1178915

\section{BIOGRAFÍAS}

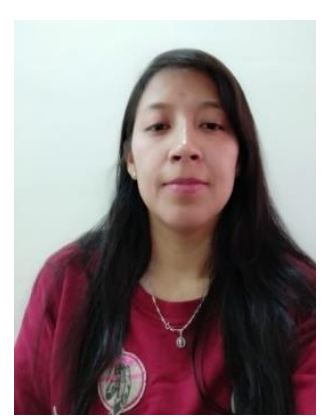

Margarita Belén Escola Brito nació en Otavalo (Ecuador), el 5 de marzo de 1993. Es Ingeniera en Ciencias Económicas y Financieras de la Escuela Politécnica Nacional (Ecuador). Conocimientos en análisis de datos. Asistente administrativa y de investigación en proyectos de desarrollo y consultorías.

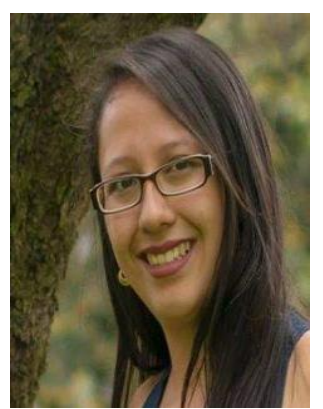

María José Palma Almendáriz nació en Quito (Ecuador), el 7 de mayo de 1993. Es Ingeniera en Ciencias Económicas y Financieras de la Escuela Politécnica Nacional (Ecuador). Conocimientos en análisis de datos. Asistente administrativa y de investigación en proyectos sobre desarrollo y consultorías. 


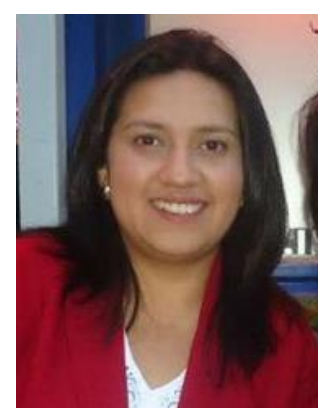

Silvia González es Doctorando de la Universidad del País Vasco (España). Master of Science in Development Evaluation and Management de la University of Antwerp (Bélgica). Ingeniera en Ciencias Económicas y Financieras de la Escuela Politécnica Nacional (EPN). Profesora titular de la EPN desde 2014. Miembro del Departamento de Economía Cuantitativa de la EPN. Ha realizado actividades de dirección y colaboración en proyectos de investigación sobre desarrollo, migración, y género; y, organización de congresos y seminarios.

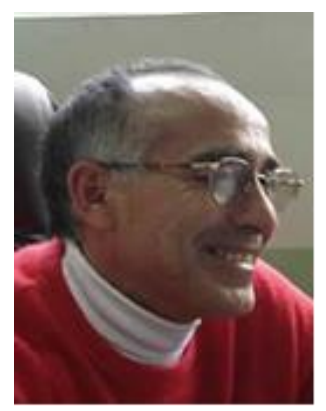

Eduardo Ávalos es Doctor en Física de la Pontificia Universidad Católica de Río de Janeiro (PUC-EPN) (Brasil) e ingeniero de la Escuela Politécnica Nacional (EPN). Coordinador de la Red Fotónica Brasil - Ecuador. Premio EPN a la Mejor Investigación 2019, y reconocimiento a mejor profesor. Fue Director de Investigación de la EPN, Decano de la Facultad de Ciencias, y Director de la Escuela de Ciencias de la Escuela Politécnica Nacional, profesor invitado en la Universidad Católica de Rio de Janeiro, ESP, UFSaoCar Sao Paulo Brasil, coordinador de la REDU. Actualmente es profesor de la Escuela Politécnica Nacional a tiempo completo y se encuentra a cargo del laboratorio de UAV's y Aplicaciones Ópticas. 
